\title{
Necrosis tubular aguda por hipercalcemia secundaria a intoxicación por vitamina D
}

\author{
Acute tubular necrosis due to hypercalcemia \\ secondary to vitamin $D$ toxicity
}

Edwin Harvey Etayo, César A. Restrepo • Manizales (Caldas)

\section{Resumen}

Los trastornos del metabolismo del calcio son considerados situaciones clínicas frecuentes. La hipercalcemia puede dar lugar a alteraciones en la función renal, su origen es principalmente el hiperparatiroidismo y las neoplasias. Nosotros reportamos el caso de una paciente quien recibió dosis altas de vitamina $\mathrm{D}$, y posteriormente hormona tiroidea y diurético tiazídico, factores que la llevaron a presentar hipercalcemia y falla renal aguda en la cual se detectó por biopsia renal la presencia de necrosis tubular aguda (Acta Med Colomb 2010; 35: 100-103).

Palabras clave: hipercalcemia, intoxicación por vitamina $D$, falla renal aguda, necrosis tubular aguda.

\begin{abstract}
Disorders of calcium metabolism are common clinical situations. Hypercalcemia, whose main causes are hyperparathyroidism and malignancy, can lead to alterations in renal function. We report the case of a female patient who received high doses of vitamin D, and afterwards thyroid hormone and thiazide diuretics. These factors caused her to develop hypercalcemia and renal failure. Acute tubular necrosis was detected by renal biopsy (Acta Med Colomb 2010; 35: 100-103).

Key words: hypercalcemia, vitamin D toxicity, acute renal failure, acute tubular necrosis.
\end{abstract}

\begin{abstract}
Dr. Edwin Harvey Etayo Ruiz: Residente 3 año Medicina Interna-Geriatria; Dr. César A. Restrepo Valencia: Internista Nefrólogo, Profesor Asociado de la Universidad de Caldas. Manizales.

Correspondencia: Dr. César A Restrepo V. Manizales-Caldas, Colombia-Sur América. Residencia: Carrera 28B \# 71 ${ }^{\mathrm{a}} 56$ Edificio los Olivos 3 piso. Teléfonos: 8813022 y 8783050. Fax: 8876692

E-Mail: caugustorv@une.net.co
\end{abstract}

\section{Introducción}

Los trastornos del metabolismo del calcio son situaciones frecuentes de la atención médica. Las causas más frecuentes de hipercalcemia son el hiperparatiroidismo primario y las neoplasias, explicando más de $90 \%$ de los casos de este trastorno electrolítico $(1,2)$.

La vitamina $\mathrm{D}$ en su forma activa $\left(1,25(\mathrm{OH})_{2} \mathrm{D}\right.$ o calcitriol) colabora en el metabolismo del calcio al optimizar su absorción intestinal y la del fósforo, pero también puede afectar el metabolismo óseo al aumentar su resorción.

La intoxicación por vitamina $\mathrm{D}$ es una causa rara de hipercalcemia, y en la mayoría de los casos inducida por el consumo de la misma sin prescripción médica. Diversos factores pueden colaborar al aumento en los niveles séricos de calcio en presencia de la administración de vitamina $D$. La complicación falla renal aguda con necrosis tubular es también una complicación infrecuente de la hipercalcemia.

\section{Exposición del caso}

Mujer de 65 años, residente en Estados Unidos de América (USA) con antecedentes de tiroidectomía total a la edad de los 34 años por carcinoma papilar de tiroides, y quien en el posoperatorio presentó hipotiroidismo e hipoparatiroidismo requiriendo desde entonces tratamiento con levotiroxina 50 ugs/día, vitamina D2 (ergocalciferol) 50.000 UI por semana y carbonato de calcio 1800 miligramos/día. Consulta a nefrología por la aparición de mareo, astenia, adinamia, náuseas, dispepsia, constipación, temblor fino en manos y cambios en el estado de ánimo. En el último control por endocrinología (dos meses antes de la aparición de los síntomas) su creatinina sérica era de $1,1 \mathrm{mg} / \mathrm{dl}$, calcio sérico 9,3 mg/dl, TSH 11,8 UI/ml, T4 libre 1,17, optándose por aumentar la dosis de vitamina D2 a 50.000 UI tres veces al día, adicionándosele calcitriol 0,5 microgramos tres veces al día, se ajustó la dosis de levotiroxina a 100 mg/día, y continuó igual dosis de calcio elemental, y de la combinación ezetimibe/simvastatina 10/20 (1 tableta diaria), la cual recibía desde hace varios años por hipercolesterolemia. Medicina Interna le había prescrito una semana antes de la consulta a nefrología hidroclorotiazida $25 \mathrm{mcg}$ /día y dieta hiposódica al detectarle cifras altas de presión arterial. En su evaluación se detectó una paciente afebril, hidratada, 
presión arterial 150/70, frecuencia cardiaca 96 por minuto, frecuencia respiratoria 16 por minuto, peso $72 \mathrm{~kg}$. En el examen físico llamó la atención sólo la presencia de temblor fino distal en ambas manos. La paciente además de los medicamentos previos y por cuenta propia había iniciado Cemtrum Silver ${ }^{\circledR} 1$ tableta/día (con 500 UI de vitamina D3 + $200 \mathrm{mg}$ de calcio elemental y $48 \mathrm{mg}$ de fósforo), Caltrate $\mathrm{D}{ }^{\circledR} 2$ tabletas/día (con $400 \mathrm{UI}$ de vitamina D3 + $600 \mathrm{mg}$ de calcio elemental), Stroven energy 1 tableta/día (con 800 UI vitamina D3 + $100 \mathrm{mg}$ de calcio elemental) y ácidos grasos omega 3, 1 tableta/día. En los paraclínicos además de los anotados previamente se detectó TSH 0.129, T4 libre 22.1, PTH intacta $10,6 \mathrm{pg} / \mathrm{ml}$, PH plasmático 7.4 con $\mathrm{HCO} 3$ de 23.2. En el examen de orina su densidad era de $1.010 \mathrm{sin}$ proteinuria y con un sedimento sin células, ecografía de cuello reportó ausencia de tiroides, endoscopia digestiva alta gastritis antral y finalmente ecografía renal con riñones de tamaño, posición y ecogenicidad normales.

Se conceptúo que la paciente cursaba con un posible cuadro de intoxicación por vitamina $\mathrm{D}$, y se le ordenó suspender la prescripción de vitamina D2, calcitriol, carbonato de calcio, diurético tiazídico y los diversos productos que inició por su cuenta. Además se redujo dosis de levotiroxina a $75 \mathrm{mcg}$ por día. Puesto que la vía oral estaba preservada se le recomendó abundante consumo de líquidos y liberar la restricción de sal prescrita por medicina interna, no se solicitaron niveles de vitamina $\mathrm{D}$ sanguíneos puesto que no se determinan rutinariamente en nuestro medio.

En control a los ocho días se evidenció mejoría de sus síntomas, y paraclínicos (Tabla 1), reiniciándose calcitriol a dosis de $0.5 \mathrm{mcg} / \mathrm{d}$ áa y carbonato de calcio $600 \mathrm{mg}$ con cada comida, con el propósito de disminuir la absorción intestinal de fósforo. Treinta días más tarde a pesar de una reducción significativa en la creatinina, sus valores continuaban altos para su edad, optándose por realizar biopsia renal percutánea, reportándose en ella necrosis tubular aguda con cambios de-

Tabla 1. Evolución de paraclínicos.

\begin{tabular}{|lcccc|}
\hline & $\begin{array}{c}\text { 2 meses } \\
\text { previos }\end{array}$ & $\begin{array}{c}\text { Día } \\
\text { cero }\end{array}$ & $\begin{array}{c}\text { 8 días } \\
\text { posteriores }\end{array}$ & $\begin{array}{c}\text { 30 días } \\
\text { posteriores }\end{array}$ \\
\hline Creatinina & $1.1 \mathrm{mg} / \mathrm{dL}$ & 2.59 & 1.14 & 1.12 \\
BUN & & $33 \mathrm{mg} / \mathrm{dL}$ & 24 & 17.2 \\
Calcio & $9.3 \mathrm{mg} / \mathrm{dL}$ & 12.08 & 9.2 & 8.4 \\
Fósforo & & 5.0 & 5.0 & 4.6 \\
TSH & $11.8 \mathrm{Ul} / \mathrm{ml}$ & 0.129 & & 9.0 \\
T4 Libre & $1.17 \mathrm{ng} / \mathrm{dL}$ & 22.1 & & 1.3 \\
PTH intacta & & $10.6 \mathrm{pg} / \mathrm{ml}$ & & \\
PH plasmático & & 7.4 & & 6.0 \\
HCO3 & & 23.2 & Negativo \\
UDensidad & & 1.010 & Sin celularidad \\
UPH & 6.0 & \\
UProteínas & & Negativas & \\
Sedimento urinario & Sin celularidad & \\
\hline U= Urinario & & & \\
\hline
\end{tabular}

generativos, regenerativos y nefritis intersticial crónica, con presencia de calcificaciones focales; la inmunoflurescencia fue negativa para inmunoglobulinas, complemento, cadenas ligeras kappa y lambda.

\section{Discusión}

La hipercalcemia puede ser el resultado de: aumento en la resorción ósea, aumento en la absorción intestinal de calcio, y disminución en la excreción urinaria de calcio.

Clásicamente se clasifican en mediadas o no por PTH (3). En las hipercalcemias secundarias a excesiva producción de PTH es característico detectar niveles bajos de fósforo gracias a la acción fosfatúrica de esta hormona.

En pacientes con valores de PTH intacta normal o bajos, los valores de fósforo sérico pueden estar normales o elevados (Figura 1). Se debe considerar en estas situaciones la posibilidad de hipercalcemia asociada a neoplasias, intoxicación por vitamina $\mathrm{D}$, desórdenes granulomatosos, consumo de medicamentos que pueden elevar el calcio sérico (tiazidas, litio, PTH sintética, vitamina A, teofilina, antiácidos con calcio), y anormalidades endocrinas como el hipertiroidismo, insuficiencia adrenal, feocromocitoma $\mathrm{y}$ acromegalia.

Entre los medicamentos que pueden elevar el calcio sérico se ha visto que para las tiazidas su mecanismo radica en su capacidad de aumentar la reabsorción del calcio en el túbulo contorneado distal (4). En pacientes con tirotoxicosis $15 \%$ a $20 \%$ presentan hipercalcemia debido a la capacidad de las hormonas tiroideas de aumentar la resorción ósea $(5,6)$.

La hipercalcemia puede generar diversas manifestaciones clínicas, las más importantes son: 1) renales: falla renal aguda, nefrocalcinosis, nefrolitiasis y diabetes insípida nefrogénica, 2) óseas: dolor óseo y artritis, 3) gastrointestinales: diarrea, constipación, náuseas vómito, anorexia, úlcera péptica e inclusive pancreatitis, 4) neuromusculares: confusión, estupor, coma, fatiga muscular, ansiedad y trastornos del estado de ánimo, 5) cardiovasculares: hipertensión, arritmias, acortamiento del intervalo QT, 6) prurito y conjuntivitis (3).

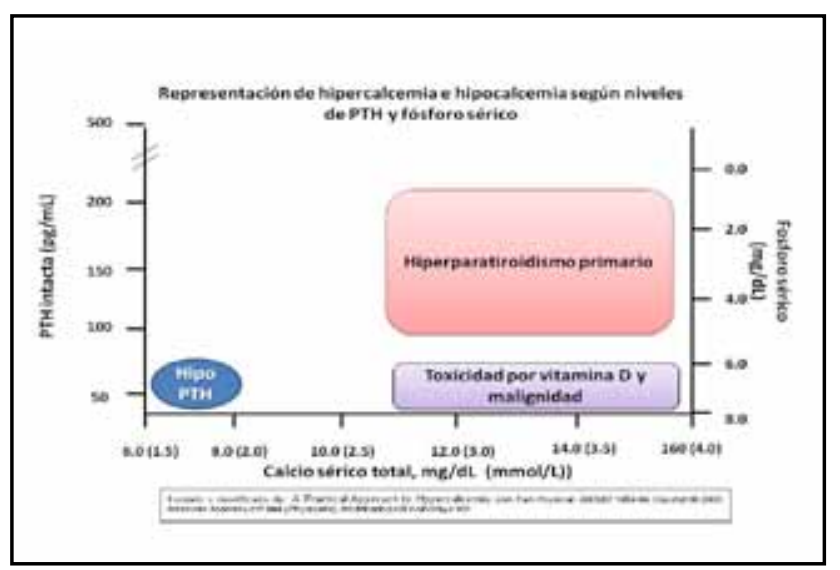

Figura 1. Correlación de valores entre PTH intacta, calcio y fósforo séricos. 
Los mecanismos que están implicados en la generación de falla renal asociado a hipercalcemia son: 1) disminución del flujo sanguíneo renal y tasa de filtración glomerular por vasoconstricción intrarrenal, efecto independiente de la hemodinámica sistémica $(7,8), 2)$ pérdidas intestinales en los pacientes que presentan diarrea, 3) deshidratación por aumento de la diuresis secundario a diabetes insípida nefrogénica.

Las manifestaciones a nivel renal incluyen necrosis tubular aguda, nefrocalcinosis, nefrolitiasis y fibrosis túbulo intersticial crónica.

La hipercalcemia secundaria a la intoxicación por vitamina $\mathrm{D}$ puede ocurrir como resultado de aporte oral exageradamente anormal de cualquiera de sus formas comerciales: vitamina D2 (ergocalciferol), D3 (colecalciferol), calcidiol $\left(25(\mathrm{OH}) \mathrm{D} 2\right.$ o D3) o calcitriol $\left(1,25(\mathrm{OH})_{2} \mathrm{D} 3\right)$. También se ha descrito cuadros de intoxicación por utilización tópica de uno de sus análogos (calcipotriol) $(9,10)$. La prescripción de dosis muy altas de vitamina $\mathrm{D}$ se ha tornado en los últimos años rutinaria, debido en parte a gran cantidad de artículos que lo estimulan, principalmente con el propósito de obtener sus beneficios extraesqueléticos, los cuales incluyen acciones antiproliferativas, prodiferenciadoras e inmunomoduladoras (11).

La dosis recomendada de vitamina D2 por el Institute of Medicine, Food Nutrition Board (FNB) es de 200 UI por día para niños y adultos menores de 50 años, 400 UI para adultos entre 51 a 70 años, y de 600 UI para mayores de 71 años (12). Para The United Kingdom Expert Group on Vitamins and Minerals (EVM) es de $25 \mathrm{mcg}$ o $1.000 \mathrm{UI} / \mathrm{día}$ de vitamina D3 (13), y para European Commission Scientific Committee on Food (SCF) 50 ugs o 2.000 UI/día de vitamina D3 (14), pero el límite de seguridad propuesto por algunos autores se ha elevado recientemente a $10.000 \mathrm{UI} /$ día de vitamina D2 $(15,16)$. Las anteriores recomendaciones tienen el inconveniente de no utilizar el mismo tipo de vitamina $\mathrm{D}$, lo cual puede dar lugar a confusiones, puesto que la vitamina $\mathrm{D} 3$, es tres veces más potente que la vitamina $\mathrm{D} 2$, mientras que el calcitriol lo es 10 veces.

El diagnóstico de intoxicación por vitamina $\mathrm{D}$ se corrobora al detectar niveles séricos de $25(\mathrm{OH}) \mathrm{D}$ mayores de $100-150 \mathrm{ng} / \mathrm{ml}(17,18)$, pero su determinación no es un procedimiento rutinario.

Se sugiere que la toxicidad por vitamina D se presenta sólo cuando su administración es superior a 10.000 UI/día, aunque la mayoría de los reportes informan dosis que van entre 100.000 a 600.000 UI por día (19-22).

La complicación generada por la intoxicación es principalmente hipercalcemia, y sólo un número muy bajo de casos llegan a falla renal aguda (23-27), siendo menos frecuente la confirmación por biopsia renal de necrosis tubular aguda (28).

En nuestra paciente en su primera atención por nefrología se detectaron síntomas típicos de hipercalcemia, la cual se confirmó por la determinación de los valores de calcio sé- rico total; no se obtuvieron niveles de albumina sérica para ajustar su valor, y tampoco fue posible obtener niveles de vitamina $\mathrm{D}$ séricos por limitaciones locales, sin embargo la evolución de la paciente fue completamente compatible con intoxicación por vitamina D. Estimamos que si 100 UI/día de vitamina D2 elevan en dos meses los niveles de $25(\mathrm{OH})$ $\mathrm{D}$ en $1 \mathrm{ng} / \mathrm{ml}, 150.000 \mathrm{UI} /$ día los llevarían a $1.500 \mathrm{ng} / \mathrm{ml}$, cifra compatible con toxicidad (15). Finalmente sin lugar a dudas se debe resaltar que la administración del diurético tiazídico y la tirotoxicosis medicamentosa contribuyeron a la hipercalcemia.

\section{Referencias}

1. Lafferty, FW. Differential diagnosis of hypercalcemia. J Bone Miner Res 1991; 6: $51-9$.

2. Burtis, WJ, Wu, TL, Insogna, KL, Stewart, AF. Humoral hypercalcemia of malignancy. Ann Intern Med 1988; 108: 454 -7.

3. Carroll M E, Shade D S. A practical approach to hypercalcemia. Am Fam Physician 2003; 67: 1959-66.

4. Gesek, FA, Friedman, PA. Mechanism of calcium transport stimulation by chlorothiazide in mouse distal collecting tubule cells. J Clin Invest 1992; 90: 429-38.

5. Burman, KD, Monchik, JM, Earll, JM, Wartofsky, L. Ionized and total plasma calcium and parathyroid hormone in hyperthyroidism. Ann Intern Med 1976; 84: 668 .

6. Iqbal AA, Burgess EH, Gallina DL, Nanes MS, Cook CB. Hypercalcemia in hyperthyroidism: patterns of serum calcium, parathyroid hormone, and 1,25-dihydroxyvitamin D3 levels during management of thyrotoxicosis. Endocr Pract 2003; 9: 517 - 21 .

7. Levi M, Ellis MA, Berl T. Control of renal hemodynamics and glomerular filtration rate in chronic hypercalcemia. J Clin Invest 1983; 71: 1624-32.

8. Chomdej B, Bell P D, Navar L G. Renal hemodynamic and autoregulatory responses to acute hypercalcemia. Am J Physiol 1977; 232: 490-6.

9. Jacobus CH, Holick MF, Shao Q, Chen TC, Holm IA, Kolodny JM, et al. Hypervitaminosis D associated with drinking milk. N Engl J Med 1992; 326: $1173-7$.

10.Hoeck, HC, Llaurberg, G, Lauberg, P. Hypercalcemic crisis after excessive topical use of a vitamin D derivative. J Intern Med 1994; 235: 281.

11. Holick M F. High prevalence of vitamin D inadequacy and implications for health. Mayo Clin Proc 2006; 81: 353-73.

12. Standing Committee on the Scientific Evaluation of Dietary Reference Intake Food and Nutrition Board, Institute of Medicine. Vitamin D. Washington DC: National Academy Press, 1999: 250-87.

13. Expert Group on Vitamins and minerals. Safe Upper Levels for vitamins and minerals. London, United Kingdom: Food Standards Agency, 2003.

14. Scientific Committee on Food. Opinion of the Scientific Committee on Food on the tolerable Upper Intake Level of vitamin D. Brussels, Belgium: European Commission, 2002.

15. Holick M F. Vitamin D ad health: evolution, biologic functions, and recommended dietary intakes for vitamin D. Clin Rev Bone Miner Metab 2009; 7: 2-19.

16. Hathcock J N, Shao A, Vieth R, Heaney R. Risk assessment for vitamin D. Am J Clin Nutr 2007; 85: 6-18.

17. Holick, MF. Vitamin D deficiency. N Engl J Med 2007; 357: 266-81.

18. Hollis B C. Circuling 25-Hydroxyvitamin D levels indicative of Vitamin D sufficiency: implications for establishing a new effective dietary intake recommendation for vitamin D. J Nutr 2005: 135: 317-22.

19. Jacobus C H, Holick M F, Shao Q, Chen T C, Holm I A, Kolodny J M, Fuleihan G E, Seely E W. Hypervitaminosis D associated with drinking milk. $N$ Engl J Med 1992; 326: 1173-7.

20. Chambellan-Tison C, Horen B, Plat-Wilson G, Moulin P, Claudet I. Severe hypercalcemia due to vitamin D intoxication. Archives de Pediatrie 2007; 14: 1328-32.

21. Barrueto F, Wang-Flores H H, Howland MA, Hoffman R S. Nelson L S. Acute vitamin D intoxication in a child. 2005; 116: 453-6.

22. Tuon F F, Nihei C H, Gryschek, R C, Seguro A C. Vitamin D intoxication: a cause oh hypercalcaemia and acute renal failure in a HIV patient. AIDS 2008; 19: $137-8$. 
23. Naik M A, Banday K A, Najar M S, Reshi A R, Bath M A. Vitamin D intoxication presenting as acute renal failure. Indian J nephrol 2008; 18: 125-6.

24. Koutkia P, Chen T C, Holick M F. Vitamin D intoxication associated with over the counter supplement. N Engl J Med 2001; 345: 66-7.

25. Titan S M, Callas S H, Uip D E, Kalil-Filho K, Galvao P C A. Acute renal failure and hypercalcemia in a athletic young man. Clin Nephrol 2009; 71: 445-7.

26. Desai B, Gokani A, Shukla A, Tampi P S. Hypercalcemic crisis-A case study of three unusual case of iatrogenic vitamin D and calcium intoxication. Bombay H J 2008; 50: 322-4.

27. Neto M M, Guimaraes F M, Ayoub F H, Viera-Neto O M, Costa J A, Dantas M. Acute renal failure and hypercalcemia. Renal Failure 2006; 28: 153-9.

28. Daher E F, Junior G B S, Queiroz A L, Ramos L M A, Santos S Q, Barreto D M S, Guimaraes A A C, Barbosa C A, Franco L M, Patrocinio R M S V.

Acute kidney injury due to anabolic steroid and vitamin supplement abuse: report of two cases and a literature review. Int Urol Nephrol 2009; 41: 717-23. 